\title{
Erratum
}

\section{Nikolaos Kanakaris, Nikolaos Tsoutseos: \\ Proximal Radio-Ulnar Synostosis at the Pin-Track Site after External Fixation of the Forearm}

\section{Eur J Trauma Emerg Surg 2007 (Published Online: February 22, 2007)}

DOI $10.1007 /$ s00068-006-5136-z

Due to technical problems one sentence in the "Patient and Method" section and a text passage in the "Discussion" section (parag. 6) were incomplete. The correct texts read as follows:

Postoperatively the arm was kept in a collar and cuff, antibiotics were continued for 3 days.

There is no completely effective or well-accepted regimen for prophylaxis against heterotopic ossifica- tion at the time of an acute injury. The effectiveness of radiation therapy in preventing heterotopic ossification after total hip arthroplasty in patients known to be at risk has been well established.

In addition, there has been an error in the assignment of the legends to figures 2,3 , and 4 . Please note the correctly assigned legends:

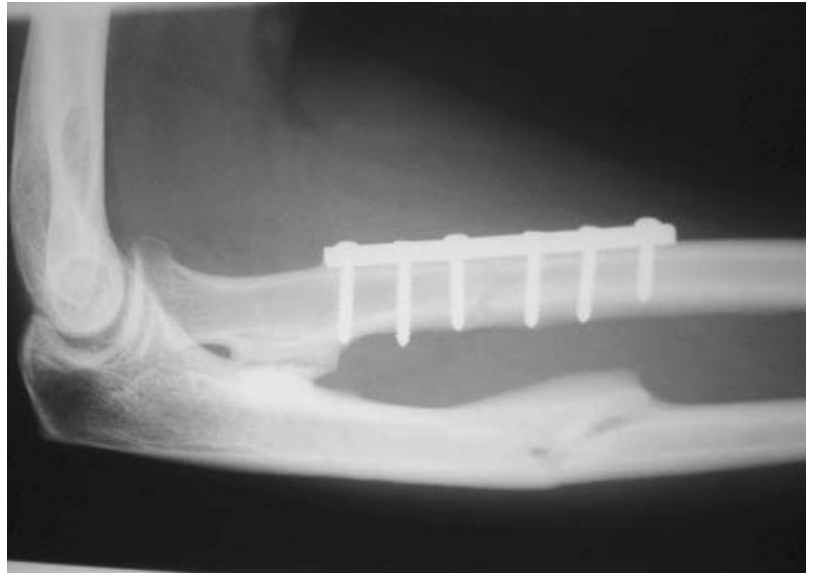

Figure 2. X-ray 5 months postoperatively. Consolidation of the synostosis. The external fixator has been removed before 2 months.

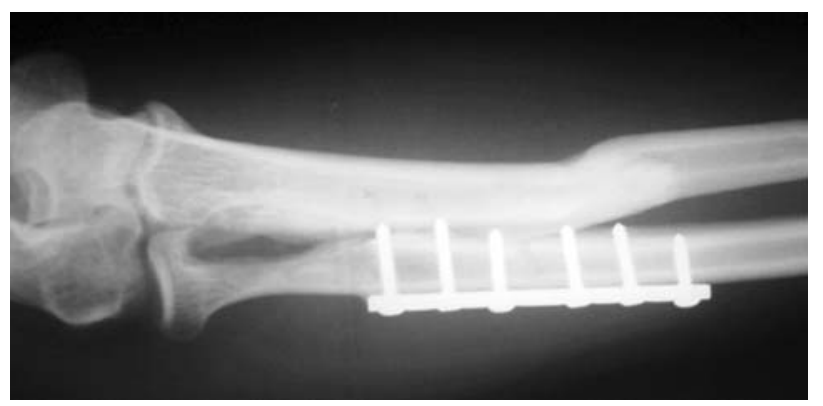

Figure 3. Postoperative X-ray of the second operation. The synostosis has been completely removed. No interposition material was used.

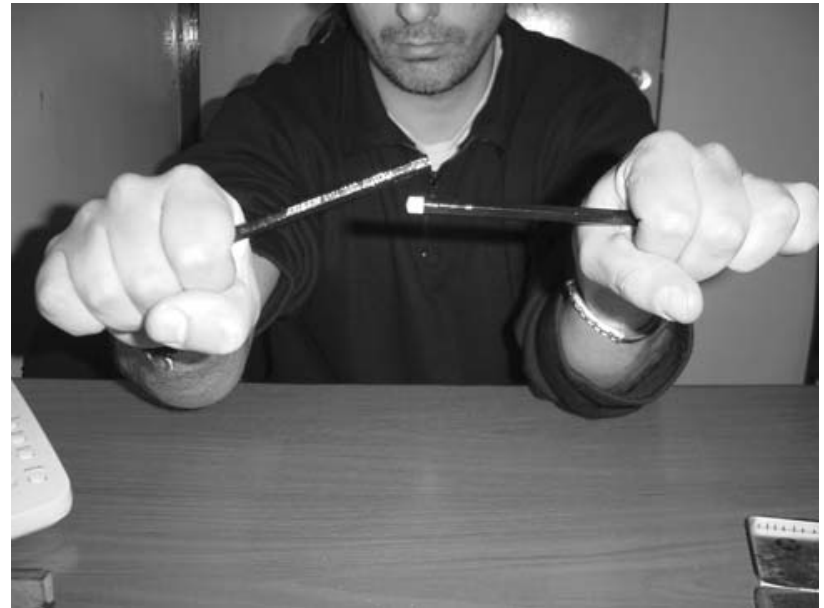

Figure 4. Seven months after the excision operation the final functional result is excellent. Forearm rotational arc is $140^{\circ}\left(60^{\circ}\right.$ of pronation). 